\title{
Article \\ Process Prediction for Compound Screws by Using Virtual Measurement and Recognizable Performance Evaluation
}

\author{
Han-Jui Chang *(D), Guang-Yi Zhang, Zhi-Ming Su and Zhong-Fa Mao \\ Key Laboratory of Intelligent Manufacturing Technology, Shantou University, Ministry of Education, \\ Shantou 515063, China; 20gyzhang1@stu.edu.cn (G.-Y.Z.); 19zmsu@stu.edu.cn (Z.-M.S.); \\ zfmao@stu.edu.cn (Z.-F.M.) \\ * Correspondence: changhj@stu.edu.cn; Tel.: +86-138-2957-9401
}

Citation: Chang, H.-J.; Zhang, G.-Y.; $\mathrm{Su}, \mathrm{Z} .-\mathrm{M}$.; Mao, Z.-F. Process Prediction for Compound Screws by Using Virtual Measurement and Recognizable Performance Evaluation. Appl. Sci. 2021, 11, 1549. https://doi.org/10.3390/ app11041549

Academic Editor: Richard (Chunhui) Yang

Received: 28 December 2020

Accepted: 4 February 2021

Published: 8 February 2021

Publisher's Note: MDPI stays neutral with regard to jurisdictional claims in published maps and institutional affiliations.

Copyright: (c) 2021 by the authors. Licensee MDPI, Basel, Switzerland. This article is an open access article distributed under the terms and conditions of the Creative Commons Attribution (CC BY) license (https:// creativecommons.org/licenses/by/ $4.0 /)$.
Featured Application: This paper presents a prediction method for predicting screw process parameters; taking crystalline and non-crystalline polymer materials as examples, when there is a lack of sufficient historical screw life data to establish a data-driven method, using various screws and polymer materials to predict optimal combination under different parameter conditions is a challenge.

Abstract: One of the important values of Industry 4.0 is to integrate people's needs into the manufacture of enhanced products, systems, and services to achieve greater levels of product customization. This paper presents a prediction method for predicting screw process parameters; taking crystalline and non-crystalline polymers as the molding material, when there is a lack of sufficient historical screw process data to establish a data-driven method, using various screws and polymer materials to predict tool life under different cutting conditions is a challenge. A screw life prediction method is proposed based on the mixed compound screw process parameters method using a dynamic iteration work. To meet the requirements of mass production, this work proposes the combined application of the automatic virtual metrology (AVM) system with the recognizable performance evaluation (RPE) program. The method predicts the injection of compound screws by extracting given cutting conditions and related process parameters characteristics from the senor data by converting sampling inspections with measurement delays from real-time and online routine inspections to automatically and quickly complete method creation production goals.

Keywords: injection molding; compounding screw; optimize molding process; automatic virtual metrology; recognizable performance

\section{Introduction}

As the global concept of Industry 4.0 continues to mature, the needs of injection molding machine users have gradually evolved from stand-alone machines to equipment automation, networking, and intelligent machines through virtual and real system integration, allowing users to have more sophisticated operational planning and effective resources distribution. This paper demonstrates the integration of the parameter-based method of machine response in simulation analysis and the material compressibility effect of molten polymer plastics. Users will be able to determine the dynamic behavior of the material more realistically when it enters the mold cavity by obtaining more accurate results from the injection pressure simulation. Through this integration method, material waste in the mold trial process will be reduced, along with a reduction of challenges faced by front-line engineers in during real process operations. In 2014, Chen et al. [1] mentioned the gravity force entering point improved filling of the cavity for the same forming time and injection pressure. The response speed of this control loop upgraded the stability of the cavity filling. 
In-line screws were designed and produced for use in injection molding machines in 1956. Along with the continuous development of new plastic materials and molding methods, screw development in recent years has been more focused on discharge volume, mixing, and homogenization. In terms of mixing, it is especially important to be able to obtain a homogeneous melt at a lower temperature level. For general plastic materials, the screw design requirement is high throughput. In the screw flow field virtual analysis, as the molten plastic is not a Newtonian fluid, due to the coexistence of both solid and liquid phases, the entire plasticization process becomes extremely complicated. There are various difficulties in establishing virtual calculation formulas for various screw designs; therefore, we tried to obtain the unprocessed design parameter values through VM methods and recognizable performance evaluation (RPE), and then used systematic and efficient experimental methods to identify the best parameter combination.

VM technology has been developed over many years, whereby all of the elements in the injection molding process are converted into virtual quantifiable values. In 2012, Cheng et al. defined [2] Virtual Metrology (VM) as a method to conjecture the manufacturing quality of a process tool based on data sensed from the process tool and without physical metrology operations. In addition, in 2016, Tieng et al. [3] mentioned VM as a method to conjecture manufacturing quality of a process tool based on data sensed from the process tool and without physical metrology operation. In other words, VM can convert sampling inspections with metrology delay into real-time and on-line total inspection.

After the calculations of the product quality and the production efficiency are completed in the virtual system, they are reflected in the physical space as recommendations for further production. In the comparison between practical injection molding and simulation analysis, the most critical implementation step is to ensure that the input data of simulation analysis is consistent with the conditions of the real-world injection processes as much as possible. However, there are many factors that can potentially cause inconsistency in subsequent comparisons, such as: the performance of the machine in terms of faster or slower mechanical response time; the characteristics in the material processing process; the data measurement method; and the geometric consistency of the product. After ensuring the correctness of these input data, the predicted results of VM are often highly consistent with the actual results and can provide users with complete calculation data in the mold cavity to facilitate the optimization and adjustment of subsequent design changes. In the comparison of injection pressure, after determining the consistency of the geometry and the position, the first issue is the construction of the material viscosity method and the acquisition of parameters; the material viscosity method needs to be able to effectively determine the temperature, shear rate, and the effect of pressure, with the more important issue being the construction of the injection machine method. The injection molding unit screw has a feeding zone, a plasticization compression zone, and a metering zone (as shown in Figure 1). The solid plastic is sent to the nozzle end by rotating the screw while drawing it back, during which time the solidified plastic changes into a molten state while being accumulated at the tip of the screw for injection. From the front end of the screw to the nozzle area, the plastic can withstand high temperatures and significant changes in compressibility, including viscosity and PVT. If this factor is included in the injection holding pressure simulation, the simulation will be better able to describe the entry conditions and produce a more accurate peak pressure forecast. 


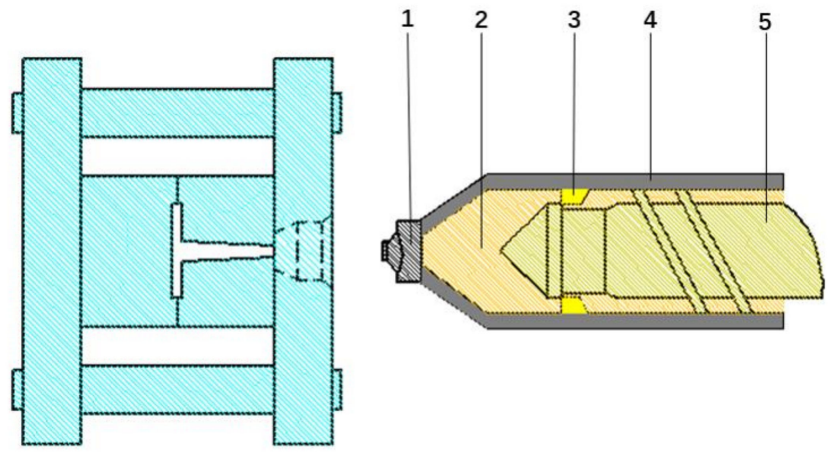

Figure 1. Example of a method of an injection machine in the injection process.

\section{Literature Review}

The differences in material compressibility are more obvious for larger changes or smaller sized products that need greater levels of precision; the VM calculation results can effectively reduce this gap. This analysis technology can currently be applied to actual customer cases to predict pressure changes during the filling process. In terms of the machine's screw motion control parameters, traditional simulations convert the motion of the screw into speed and pressure that is simply applied to the molten plastic, which greatly oversimplifies the flow behavior of the plastic. Taking a closed loop hydraulic press as an example: in the actual injection stage, to eliminate the difference between the measured speed and the injection speed input by the injection molding machine engineers, the machine adjusts the controller valve proportionally to increase or decrease the rate of advance.

In 2019, Lin et al. [4] developed a predictive maintenance goal to determine when a target device (TD) is in a poor condition and is actually about to break down, so that the TD can received timely maintenance to avoid unexpected downtime. This not only improves the availability and manufacturing quality of tools, but also reduces the additional cost of excessive maintenance in preventive maintenance strategies. In 2017, Chiu et al. [5] proposed that the main purpose of predictive maintenance (PdM) is to reduce unplanned downtime, thereby increasing productivity and reducing production costs. In 2019, Yang et al. [6] mentioned that generally speaking, it is not easy to collect enough early samples. This issue delays the preparation of tool diagnostic methods. The purpose of their article was to propose a progressive refresh method for operating and refreshing tool diagnostic methods. In addition, a sample expansion method was proposed to reducing method time and improve the accuracy of tool status classification. In 2017, Lin et al. [7] proposed a design based on the implementation of the Internet of Things (IoT), Cloud Computing (CC), Big Data Analysis (BDA), Cyber-Physical System (CPS), and the predictive technology manufacturing intelligent platform Advanced Manufacturing Cloud of Things (AMCOT) to achieve increased production with a confirmed production method. In 2017, Hung et al. [8] provided a feasible systematic solution to effectively correct the MC related limitations of the existing AVM system, as the existing VM-related literature mainly focused on the development of the VM method. In 2017, Yang et al. [9] proposed a tool cyber-physical prediction (TCPP) method and a hybrid dynamic neural network (HDNN) method. By integrating the theoretical maximum tool (cutter) life and actual tool (cutter) wear sensing functions, this solution enables users to build and use cloud methods that can be applied to factory machines. In 2015, Yang et al. [10] presented a novel idea for VM use, which predicts wheel machining automation quality based on process data collected from machine tools, and slightly supplements the actual measurement data. The authors proposed the use of AVM technology, which has been successfully applied to high-tech industries such as semiconductors, displays, and solar cells. In 2016, Cheng et al. [11] noted that whenever the VM accuracy deteriorates, it cannot adaptively increase the default sampling rate in the intelligent sampling decision (ISD) method; as a result, it takes more time to collect enough samples to improve the accuracy of the VM. When the VM accuracy is 
always maintained in good condition, it cannot automatically reduce the default sampling rate in the ISD, which may cause unnecessary waste. In 2019, Chen et al. [12] used VM to monitor the electrical discharge machining (EDM) process; a probe with a very high sampling rate is required to obtain the voltage and current signals of the electrode, and so a large amount of sensor data is generated. When extracting features from the raw sensor data, there can be big data processing problems associated with the sensor data. When there is interaction between variables, the influence is also greater than the impact of the original device/variable, and the original key-variable search algorithm (KSA) solution may not be able to correctly find the root cause. In 2018, Lin et al. [13] researched key equipment/variables and other equipment, and to make up for any deficiencies, developed the interaction-effect search algorithm (IESA). The IESA method can not only identify the existence of interactions, but can also determine the thresholds of key variables that lead to such interactions.

In 2017, Cheng et al. [14] provided users with a KSA solution that delivers a quick and effective way to determine the root cause of the output. However, when a process constitutes equipment or a process that has multiple pieces of equipment with the same defect density, if the root cause lies in the above-mentioned pieces of equipment, the KSA cannot correctly identify it. This is the so-called blind stage problem. The purpose of this article was to propose a blind-stage search algorithm to solve the blind-stage problem. In 2016, Yang et al. [15] proposed a tool life prediction method based on a hybrid tool wear method with a dynamic neural network. This method predicts tool wear after extracting the cutting conditions and related tool wear features from the sensing segment data. In 2015, Tieng et al. [16] researched the use of VM to measure the machining accuracy of machine tools. As shown on the left side of Figure 1, the AVM server [6] requires process data, including sensor data and processing parameters, as input to predict the processing accuracy. As processing operations usually manifest severe vibrations and loud noises, the raw data collected from the sensors attached to the machine tool have a low signal-to-noise (S/N) ratio, which affects the prediction accuracy of the VM. In 2015, Cheng et al. [17] proposed an AMCoT platform, which cannot only achieve the goals of Industry 4.0, but also achieve the goal of zero defects when applied to AVM technology. In 2019, Yang et al. [18] proposed using an in situ metering (ISM) module and an enhanced AVM system to create an intelligent metrology architecture. The ISM module uses a coaxial camera and a pyrometer to extract the melting pool features. The AVM system is improved by a feature selection method to solve the problem of limited sample sizes of quality method parts. In 2018, Tieng et al. [19] measured long metrology delays and severed component deformations. To overcome these two challenges that are related to the aerospace industry, the article recommends the use of an AVM system to successfully convert sample inspections that have metrological delays into real-time and online general inspections. This article discussed the use of a deformation fusion (DF) method to deal with component deformation issues. In 2016, Tieng et al. [3] required a screw design unit with MC capability to be developed with a high degree of rapid response capability to better accurately manufacture various types of wheels where processing conditions are changing. To meet the requirements of MC production, this work proposed the application of an AVM system along with a target value adjustment (TVA) method. In 2016, Cheng et al. [20] mentioned that when encountering a yield loss, the traditional yield method is to collect all production-related data for big data analysis to find the root cause that affects the output and then remedy it. This article proposed a method of key-variable search algorithms (KSAs); the inputs of the KSA method includes production routes, process data, online data, defects, and the final inspection results. The outputs of the KSA method are the search results and their corresponding reliance indices. In 2015, Hsieh et al. [21] noted how AVM technology had been applied to various industries to convert the offline sampling inspection with metrology delay into online and real-time total inspection. With the assistance of AVM, each workpiece of carbon fiber products can be inspected to make sure that a comprehensive quality inspection has taken place. In 2017-2018, Chang [22-24] mentioned how the concept of the interaction 
effect is explained in physics as being when two or more independent variables exist in an experimental study, and how the effect of one of the independent variables exhibits an alternating phenomenon on the other independent variable. The true effect of some factors is changed by another factor. The VSM method in Taguchi's method is used to evaluate this interaction effect. In 2019, Chang [25] reviewed various methods to evaluate the performance of five-axis machine tools through identification and multi-type comparison, with the RPE being the most practical. RPE is currently one of the research methods that can obtain accurate reference data with both a quantitative and identifying approach and is one of the evaluation methods for multi-type five-axis machine tool methods. Based on the interface of RPE and IT level distribution in general mechanical design change, this paper attempted to introduce fuzzy theory to obtain excellent research results. This study calculated the attribution level of the tested items. With regards to the conflicts in the re-evaluation of the performance of various types of five-axis machine tools, the research method provided a direct differentiation and defuzzification of the attribution reduction interval, where the predicted results can be directly judged and evaluated. In 2020, Chang [26] quantified the interactions between the warpage directions of glass fibers. This method provided reference data along with a simple method to determine whether it affects the mold, depending on whether glass fiber was added, and the holding pressure time conditions.

Regarding the compression effect on the screw at the front end of the barrel, during the injection and holding process in the virtual simulation, the material in the barrel and nozzle of the injection machine displays transient compression behavior; the integrated machine response parameter method with the simulation of the material compressibility effect of the polymer molten plastic injection pressure is rarely mentioned in the related literature. The traditional VM technology only considers a single phase change in the machine settings, so the prediction of the difference in molten plastic pressures will be different from the experimental values. Considering the machine integration and the barrel compression effect, the injection pressure prediction curve could be greatly improved, whereby the simulated predicted value is closer to the actual value, and the machine integration prediction is closer to the actual settings. Data is key to this process. In 2020, Fanny Castéran, Ruben Ibanez, Clara Argerich, Karim Delage, Francisco Chinesta, and Philippe Cassagnau [27] tried to combine a classical 1D twin-screw extrusion model with machine learning techniques to obtain accurate predictions of a complex system despite few data. The used machine learning techniques with low data limit were used as a correction for the simulation.

\section{Research Purpose}

With the advent of the IoT and the CPS era, Industry 4.0 redefines the industrial manufacturing system into a fully automated scenario. The digital, intelligent, and customizable features of this industrial development have pushed traditional manufacturing technology from mass production to deep-rooted mass customization. Although the concept of mass customization is not new, this idea has been emphasized again in Industry 4.0 as customers have returned to this core value. One of the core values of Industry 4.0 is to integrate people's needs into the manufacturing of enhanced products, systems, and services to achieve greater personalization of customized products. With Industry 4.0, the manufacturing industry will undergo further changes that will benefit customers. Generally speaking, the birth of IoT and CPS has raised data collection and communication technology to a new level so that it can respond to customer needs faster. Industrial manufacturers can effectively build relationships with end customers by combining the flexibility and personalization of real-time customization. Mass customization also encompasses the concept of make to order, as only after the manufacturer knows the customer's needs will production be carried out. Customers or end users can easily determine certain functions or personal attributes of unique products or services they really want through the website portals, so that customers, manufacturers, and devices are seamlessly connected through the IoT and 
CPS to closely interact with each other. This is a win-win situation for all participants in the modern manufacturing relationship.

Regarding the VM real-time full inspection method, this was proposed in order to be able to infer product quality and manufacturing processing data without using physical measurements. In other words, VM can convert sampling inspections and metrology delay into real-time and online total inspection. To automate the VM technology, an AVM system was developed. AVM is a promising technology that meets the requirements for zero defects, and this technique allows for metrology delays. Generally, in the production process of traditional screws, it is usually necessary to make corrections after manufacturing, and then redefine the mixing conditions for each polymer material in the screw after each experiment. The workpiece then has product correction tracking implemented in certain stages or screw grooves. By using a unique RPE value, various parameters of each screw workpiece during the screw groove process can be collected for use in intelligent manufacturing applications, such as AVM in intelligent yield improvement and engineering data analysis applications. However, this situation cannot be applied to workpiece products of mixed condition screw grooves that continuously have their slope changed, because it is impossible to define the groove size of the continuous production of mixed screws. To solve this undefinable problem, this study defines a correction method with the combined AVM and RPE methods, which is expressed as production data backtracking mechanism for the continuous molten polymer production of raw materials in the screw groove. This mechanism collects the production data of all workpieces and sends them to the AVM server to perform a comprehensive inspection on the continuous production of molten polymer raw materials in the screw groove.

In the simulation analysis, the user manipulates the filling speed and pressure response settings of the machine and uses the experimental method to identify the machine integration method; real machine conditions can then be incorporated into the VM analysis for consideration. As traditional simulation technology analysis can only show single-stage flow rate predictions, the injection rate can be identified through machine integration, which can obtain the changing flow rate behavior that is closer to the real machines conditions, along with the behavior of the flow rate delay in the initial stage. The speed of machine response follows a non-linear control method, so inserting the AVM and RPE analyses into the simulation is an issue that users will encounter under given conditions (as shown in Figure 2).

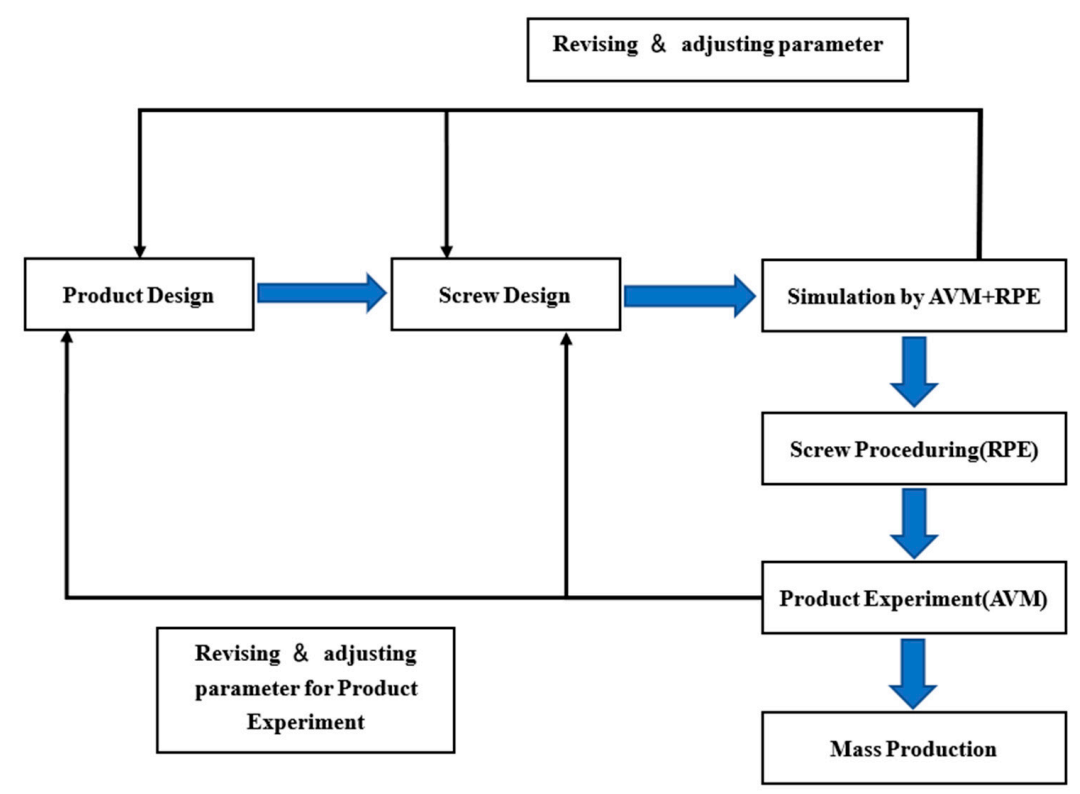

Figure 2. Flowchart of AVM+RPE method. 


\section{Automatic Virtual Metrology Control Method}

The AVM method and operational process data are shown in Figure 2. The method process data are adjusted through the AVM method, and then used to build various VM methods. The data used are from the newly manufactured screw process parameter prediction method. The operating principle of the AVM method mainly includes the RPE check of the operation sample, along with the grouping of method samples (process data) and adjustment of their target values. A set of deterministic samples are defined in AVM, which are then extracted from injection molding machine parameters and used to automatically calculate the target values. The required sample size must be defined, as it is used it to determine the number of conversions of the AVM method; experts recommend about 3-5 per group. The target value (TV) of the operational sample is determined by the average measurement value, as shown in Formula (1), where $y_{j}$ represents the actual measurement value of the $j$ number of elements, and $n=$ the size of the TV [3].

The AVM system converts sampling inspections with measurement delays into realtime and online total inspections, while the TVA method aims to strengthen the adaptive customization function of the AVM to accomplish the MC production goals automatically and quickly which are shown in Figure 3. To meet the requirements of MC production, this paper proposes to apply the AVM system together with the target value method.

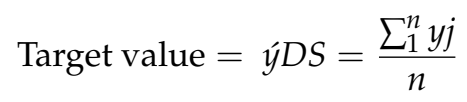

\section{$\times$ Adjusted Metrology with TVA - Metrology of Type B}
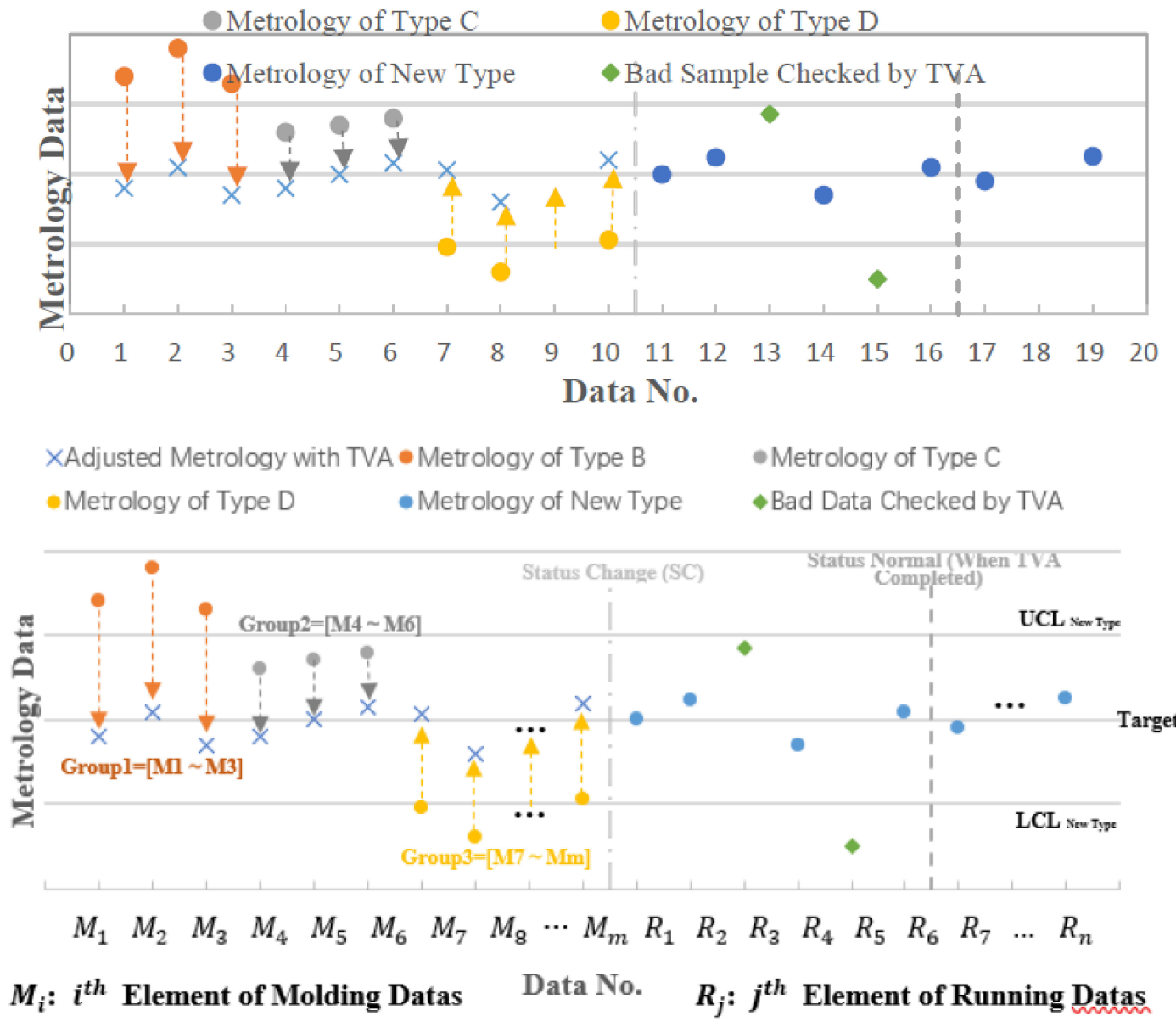

Figure 3. Operating scenarios of method and running data of the AVM method. 
It is necessary to reconfigure this to ensure the measurement accuracy of both the functions of the production tool and the compression ratio; the first few tests after the polymer material is changed might be unstable until measurement accuracy is achieved.

To ensure the measurement quality after operations, the AVM system first conducts a quality inspection that is used to calculate the incremental difference $(\delta)$ between adjacent samples; this inspection determines whether $\delta$ is greater than the value of $\delta\left(\delta_{\mathrm{T}}\right)$, where $\delta_{\mathrm{T}}$ is calculated using the specification tolerance and the tolerance ratio $(\alpha)$, defined in Formula (2).

$$
\delta_{\mathrm{T}}=\alpha * \mid \mathrm{UCL}_{\mathrm{New}} \text { Type }-\mathrm{LCL}_{\mathrm{New}} \text { Type } \mid
$$

The default specification includes the upper control limit (UCL) and lower control limit (LCL) that can be set with new samples. After the new measurement sample is checked, it will be added to the deterministic sample. It takes a lot of time and cost to collect a sufficient number of specific types of samples to build a VM method; it is possible to create a method by using multiple types of samples, thus reducing the time required for the MC process. However, the problem of insufficient causality between measurement values and different types of samples must be resolved. To distinguish different types of samples with different TVs, it is necessary to group the method samples. As shown on the left side of Figure 2, the method sample contains three types of metrics with different TVs. They can be sorted into ascending order by applying Formula (3) to obtain $\mathrm{y}_{\mathrm{M}}$, where $\mathrm{y}_{\mathrm{i}}$ represents is the i number of elements of the method sample, $m=S I Z E y_{M}=$ the size of the method sample.

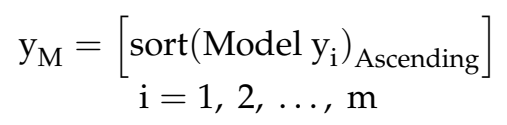

\section{Illustrative Example of Injection Screw Machining Process}

This section gives an example of the TVA method's operational process. Before the actual processing occurs, the AVM and RPE methods mentioned in this paper were applied, so the AVM-RPE methods were used with the barrel screw process to calculate five different types of screw groove and special design issues. By applying the method to the barrel screw, key features that reflect the customization changes can be determined; this is advantageous as screw grooves without relevant key features can be used to solve virtual identification issues.

Generally speaking, the design process of barrel screws can be roughly divided into three categories according to the different polymers used: (1) fast melting polymer such as PMMA; (2) medium melting polymer such as PS, ABS, and SAN; and (3) low melting polymer such as PVC-U and PVC-C, examples of which are shown in Figure 4, Figure 5, and Figure 6, respectively. For illustrative convenience, this paper uses fast melting polymer PMMA to introduce this type of barrel screw design process as a batch processing example for the machine center screw design unit. In this example, the width of the solid bed as processed by the screw groove space design is used as the inspection item. For the purpose of illustration, the recommended length $(\mathrm{mm})$ of each slot width of the feeding section, the charging section, and the compression section are described to provide a true measurement for accuracy checking.

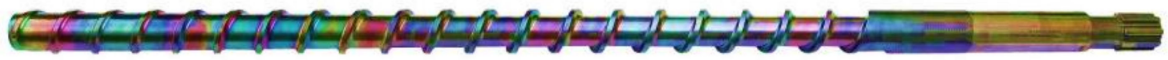

Figure 4. Experiment barrel screw for fast melting polymer design.

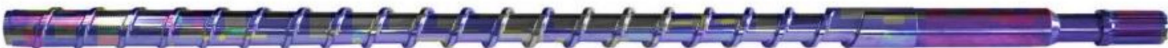

Figure 5. Experiment barrel screw for medium melting polymer design. 
Figure 6. Experiment barrel screw for low melting polymer design.

To prove the flexibility and effectiveness of the TVA method, three types of thermaladaptive PMMA polymer alloy screws were processed in batches in the injection machine's screw manufacturing unit. In this example, the average screw length (ASL) of the processed screw was used as the inspection item [28,29]. As Figure 7 compares the difference of molten plastic prediction under various calculation conditions.

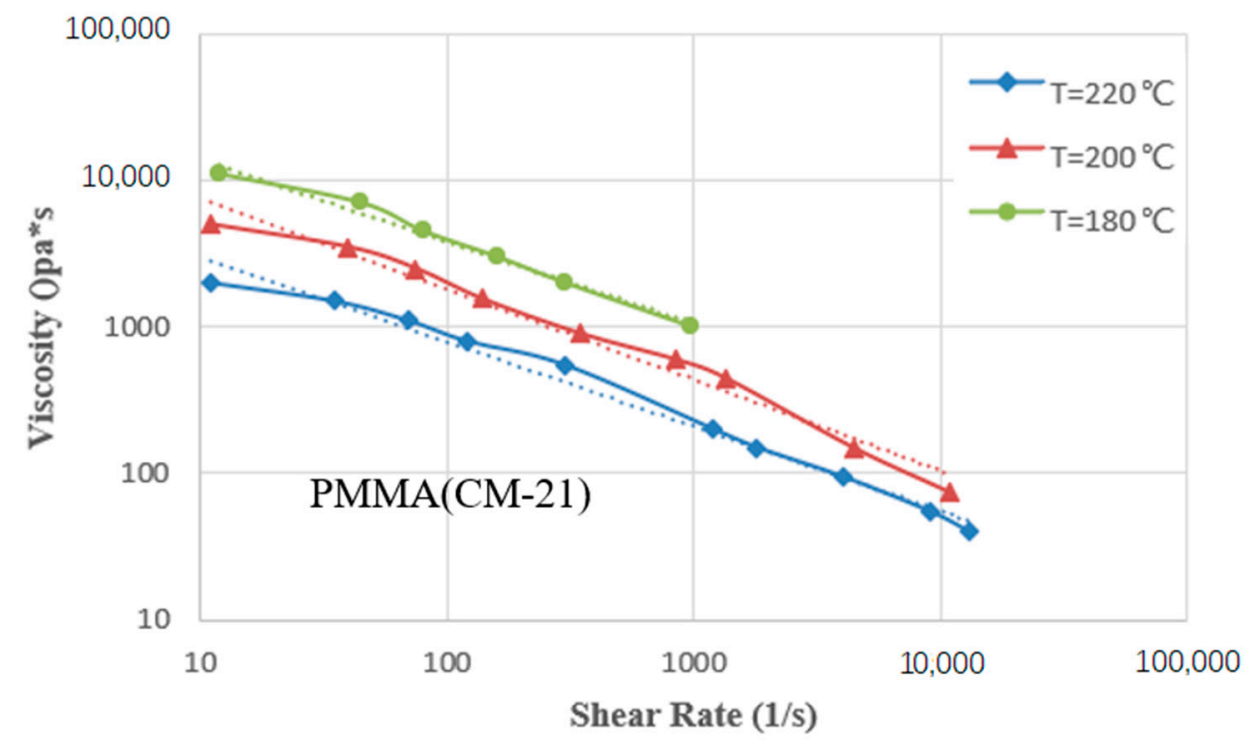

Figure 7. Temperature information for PMMA material design.

Calculation examples and procedures

$\varnothing 40, \frac{\mathrm{L}}{\mathrm{D}}=20, \mathrm{~L}_{1}=9 \mathrm{D}, \mathrm{L}_{2}=8 \mathrm{D}, \mathrm{L}_{3}=3 \mathrm{D}$,

$\mathrm{h}_{1}=6.9 \mathrm{~mm}, \mathrm{~h}_{3}=3.1 \mathrm{~mm}, \mathrm{~s}=40 \mathrm{~mm}, \mathrm{e}=4 \mathrm{~mm}$.

$\mathrm{n}=100 \mathrm{rpm}$, screw front pressure $57 \mathrm{kgf} / \mathrm{cm}^{2}$, Feeding temperature $75{ }^{\circ} \mathrm{C}$, Barrel temperature $220^{\circ} \mathrm{C}, 215^{\circ} \mathrm{C}, 210^{\circ} \mathrm{C}, 205^{\circ} \mathrm{C}$.

$\mathrm{h}=$ Starting depth of the gradual groove

$\mathrm{h}_{1}=\mathrm{L}_{1}$ Starting depth of the gradual groove

$\mathrm{h}_{2}=\mathrm{L}_{2}$ Starting depth of the gradual groove

$\mathrm{s}=$ Injection stroke

$\mathrm{e}=$ Axial thread width

$\mathrm{n}=$ Rotating speed (rpm)

For PMMA material, the properties are as follows:

$\mathrm{Tg}=100{ }^{\circ} \mathrm{C}, \mathrm{Cs}=2174 \mathrm{~J} / \mathrm{kg}{ }^{\circ} \mathrm{C}, \mathrm{Cm}=2174 \mathrm{~J} / \mathrm{kg}{ }^{\circ} \mathrm{C}, \rho_{\mathrm{s}}=1160 \mathrm{~g} / \mathrm{cm}^{3}$, $\rho_{\mathrm{m}}=1040 \mathrm{~g} / \mathrm{cm}^{3}, \mathrm{~km}=0.255 \mathrm{w} / \mathrm{m}^{\circ} \mathrm{C}$. Charging volume of melting zone, $\mathrm{Q}=17.22 \mathrm{~cm} / \mathrm{sec}$

$\mathrm{T}_{\mathrm{g}}=$ Glass transition temperature

$\mathrm{C}_{\mathrm{S}}=$ Specific heat of solid phase raw material

$\mathrm{C}_{\mathrm{n}}=$ Specific heat of liquid phase raw material

$\mathrm{Z}_{\mathrm{T}}=$ Total length of screw melting zone required polymer materials

$\mathrm{W}=$ Screw groove width

$\mathrm{X}_{1}=$ Began solid material bed weight

$\mathrm{G}=$ Mass flow rate for stable extrusion

$\Psi=$ Melting index, PMMA is $\Psi=8.38 \times 10^{-3}$

$\mathrm{H}=$ Starting depth of gradual screw groove

$\mathrm{A}=$ Gradient

1. Helix angle $\theta=\tan ^{-1} \frac{S}{\pi D}=\tan ^{-1} \frac{40}{\pi 40} 17.66^{\circ}$

2. Average screw length 
Feeding Zone $Z_{1}=\frac{\pi\left(D-h_{1}\right)}{\cos \theta}=\frac{\pi(40-6.9)}{\cos \left(17.66^{\circ}\right)}=109.1 \mathrm{~mm} / \mathrm{pitch}$

Melting Zone $Z_{3}=\frac{\pi\left(D-h_{3}\right)}{\cos \theta}=\frac{\pi(40-3.1)}{\cos \left(17.66^{\circ}\right)}=121.7 \mathrm{~mm} /$ pitch

Compression Zone $Z_{2}=\frac{Z_{1}+Z_{3}}{2}=115.4 \mathrm{~mm} /$ pitch

Compression Zone total length $Z_{A}=8 \times Z_{2}=923 \mathrm{~mm}$

Gradient $A=\frac{A_{1}-A_{3}}{Z_{A}}=\frac{6.9-3.1}{923}=4.1 \times 10^{-3}$

Groove width $W=(s-e) \cos \theta=(40-4) \times \cos \left(17.66^{\circ}\right)=34.3 \mathrm{~mm}$

\section{Theoretical Process}

Here, theoretical process equations are the results of the solid bed width profile for a tapered channel, which on the basis of the research of Zehev Tadmor [28,29] which theoretical model as (4) in the following,

$$
\frac{X}{W}=\frac{X_{0}}{W}\left\{\frac{\Psi}{A}-\frac{\left(\frac{\Psi}{A}-1\right)}{\left[1-\frac{Z}{Z_{T}} \frac{A}{\Psi}\left(2-\frac{A}{\Psi}\right)\right]^{\frac{1}{2}}}\right\}^{2}
$$

Defined as being vectorless in the above formula, its physical meaning describes the ratio of the beginning melting rate per unit length to the solid mass flow rate per unit depth. If it starts to melt at the beginning, $X_{1}$ must be replaced with $W$. If melting starts somewhere in the part, a set of similar equations can be derived, in which case the conditions in the above equation are different. This special case is melting in the barrel channel, which is similar to the proposed derivation. Assuming that the melting starts in the feeding section, the solid bed width $X$ in the feeding section can be defined as:

$$
\frac{X}{W}=\frac{X_{0}}{W}\left[1-\frac{\varnothing W^{1 / 2}}{2 Q} Z\right]^{2}
$$

$\varnothing: \mathrm{g} / \mathrm{sec} \cdot \mathrm{cm}^{1.5}, Q=\mathrm{g} / \mathrm{sec}, W: \mathrm{cm}, Z: \mathrm{cm}$

$X_{0}=W\left(\right.$ melting begin, $\left.X_{0}=W\right)$

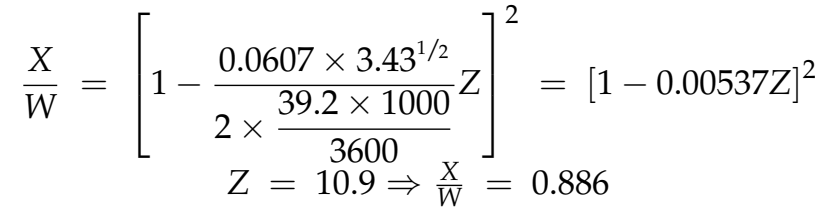

If $Z=21.8 \Rightarrow \frac{X}{W}=0.78$

$$
\begin{aligned}
Z_{T}= & \frac{1}{0.00537}=186.2 \mathrm{~cm} \\
& \frac{186.2}{10.9}=17 D
\end{aligned}
$$

Width of the solid bed in compression section X:

If melting starts from the beginning of the compression section. Then the starting solid bed width is $X_{0}=W=3.43$

If the melting pool is formed in $2 \mathrm{D}$ before the compression section, then 


$$
\begin{aligned}
& \begin{array}{c}
\frac{X_{0}}{W}=0.78 \\
\Psi=\frac{\Phi W^{1 / 2}}{\frac{Q}{h_{1}}(0.78)^{1 / 2}}=8.38 \times 10^{-3}
\end{array} \\
& Z_{T}=\frac{h_{1}}{\Psi}\left(2-\frac{A}{\Psi}\right) \\
& \Psi=7.4 \times 10^{-3} \\
& Z_{T}=\frac{0.69}{7.4 \times 10^{-3}}\left(2-\frac{4.1 \times 10^{-3}}{7.4 \times 10^{-3}}\right)=134.8 \mathrm{~cm} \\
& \frac{Z_{T}}{Z_{2}}=\frac{134.8}{11.54}=11.7 \mathrm{D} \text { All can be plasticized } \\
& \Psi=8.38 \times 10^{-3} \\
& Z_{T}=\frac{0.69}{8.38 \times 10^{-3}}\left(2-\frac{4.1 \times 10^{-3}}{8.38 \times 10^{-3}}\right)=124.4 \mathrm{~cm} \\
& \frac{Z_{T}}{Z_{2}}=\frac{124.4}{11.54}=10.8 \mathrm{D} \text { All can be plasticized }
\end{aligned}
$$

The solid bed/groove width at the groove length $\mathrm{Z}$ in the compression section can be calculated using the following formula [28,29]:

$$
\begin{aligned}
& \frac{X}{W}=\frac{X_{0}}{W}\left\{\frac{\Psi}{A}-\frac{\left(\frac{\Psi}{A}-1\right)}{\left[1-\frac{Z}{Z_{T}} \frac{A}{\Psi}\left(2-\frac{A}{\Psi}\right)\right]^{\frac{1}{2}}}\right\}^{2} \\
& \frac{X_{0}}{W}=0.78, \Psi=8.38 \times 10^{-3}, Z_{T}=124.4 \mathrm{~cm} \\
& \frac{\Psi}{A}=\frac{8.38 \times 10^{-3}}{4.1 \times 10^{-3}}=2.04 \\
& \frac{X}{W}=0.78\left\{2.04-\frac{(2.04-1)}{\left[1-\frac{Z}{Z_{T}} \times \frac{1}{2.04}\left(2-\frac{1}{2.04}\right)\right]^{\frac{1}{2}}}\right\}^{2} \\
& =0.78\left\{2.04-\frac{1.04}{\left[1-0.74 \frac{Z}{Z_{T}}\right]^{\frac{1}{2}}}\right\}^{2}
\end{aligned}
$$

In this example, the screw design unit reflects the change of the screw design unit with the TV adopted by the AVM method. Therefore, the reason and key features of the TV change can be found in the design process; the quantitative production issue of the TV change can be supplemented by the application of key features. A total of five screw groove samples were used here, from $\frac{Z}{Z_{T}}=0-0.9$, which included five key features. We can deduce:

$$
\begin{aligned}
\frac{Z}{Z_{T}} & =0 \Rightarrow \frac{X}{W}=0.78 \\
\frac{Z}{Z_{T}} & =0.1 \Rightarrow \frac{X}{W}=0.718 \\
\frac{Z}{Z_{T}} & =0.2 \Rightarrow \frac{X}{W}=0.65
\end{aligned}
$$




$$
\begin{aligned}
& \frac{Z}{Z_{T}}=0.5 \Rightarrow \frac{X}{W}=0.415 \\
& \frac{Z}{Z_{T}}=0.9 \Rightarrow \frac{X}{W}=0.045
\end{aligned}
$$

The above process proves the flexibility and effectiveness of the AVM system method. From the above conclusions, we can obtain: when $\frac{Z}{Z_{T}}=0.5$, and $\frac{X}{W}=0.415$, the relationship in the screw groove $L / D$ ratio diagram converges. This means that at this time, the $\mathrm{L} / \mathrm{D}$ ratio will have a relatively similar design basis, and there will be no large differences between the groove width and solid material bed weight. However, to determine whether a better melting and plasticizing effect can be obtained in actual production, it is necessary to verify the RPE method in a real process. Five different types of groove and space design problems were calculated through the AVM method. Through the application of the method to the barrel screw, key features that reflect the customization changes could be determined, the benefit being that screw grooves without relevant key features can be used to solve virtual identification issues.

For illustrative purposes which are shown in Figure 8, SD- $\sigma$ and S/N ratios were used as algorithms for method interpretation. Given the predicted value, the actual value, and the sample size, the SD- $\sigma$ and $\mathrm{S} / \mathrm{N}$ ratios represent the verification efficiency of the screw melting groove. In this example, real measurements of the accuracy check are used, including data collection, groove space, and signal. After segmentation, data cleaning, and feature extraction, the three recognizable signals included: full range, average and standard deviation; and the two interpretation signals included full range/average, and the selected signal features of three times standard deviation/average values which are shown in Table 1.

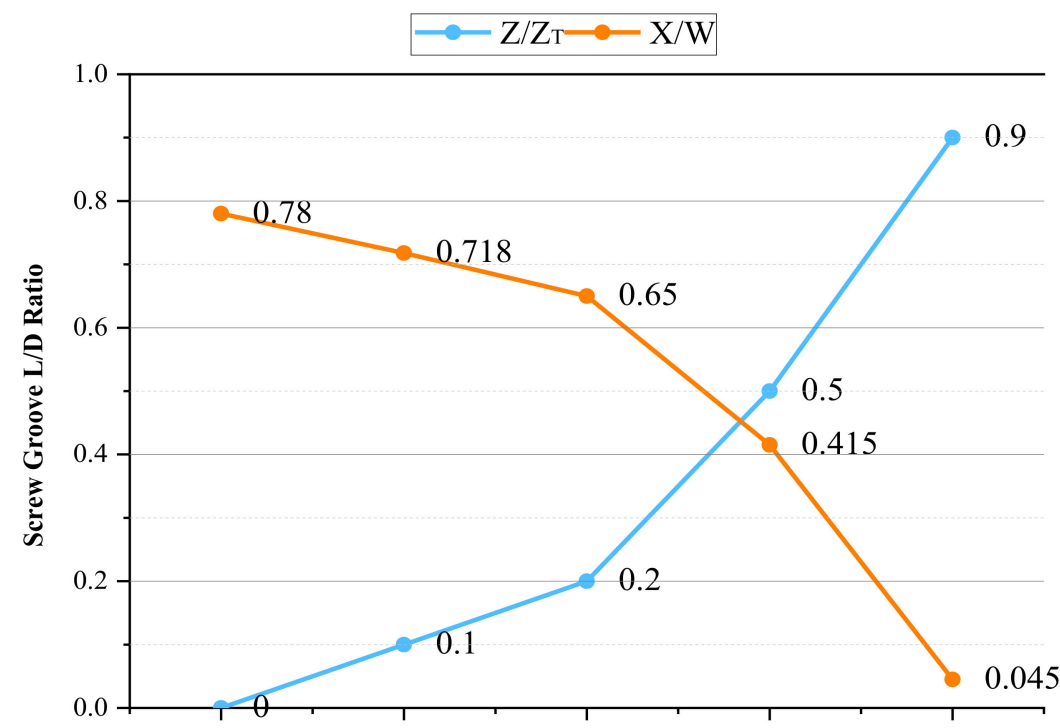

$\mathrm{Z} / \mathbf{Z}_{\mathrm{T}}=$ Average screw length/ Total length of screw melting zone required polymer materials $\mathrm{X} / \mathrm{W}=$ Groove width $/$ Solid bed width

Figure 8. Relationship of screw groove L/D ratio.

Table 1. Relationship of screw groove L/D ratio.

\begin{tabular}{ccccc}
\hline Item & Inject Time (sec) & Charging Time (sec) & R-Inject Time (sec) & R-Charging Time (sec) \\
\hline Average & 0.1282 & 11.5955 & 0.1287 & 11.8233 \\
SD- $\sigma$ & 0.0038 & 0.5353 & 0.0034 & 0.3442 \\
Range & 0.0100 & 2.2300 & 0.0100 & 1.6200 \\
R/X bar & 0.0780 & 0.1923 & 0.0777 & 0.1370 \\
S $/$ X-bar & 0.0901 & 0.1385 & 0.0793 & 0.0873 \\
SN Ratio & 30.45 & 26.71 & 31.56 & 30.72 \\
\hline
\end{tabular}


This example includes two different sets of actual injection molding sample parameters to verify the VM method in terms of processing the width of the groove cavity, which are shown in Figure 9; these parameters include: inject time (sec) and charging time (sec). There are reasons to use these two sets of sample parameters, with the main reason being to predict the size of the screw melting groove. The best verification method is charging time (sec) performance, and the charging time (sec) time length can be used as one of the verification parameters. In addition to the screw melting efficiency, if the polymer material is completely melted and mixed, its injection behavior must be quite smooth, and the inclusion of injection time (sec) in the sample parameter is also well-founded. The first 105 groups of sample parameters were used to build the VM method, and these test samples were used to evaluate the accuracy of the estimation.

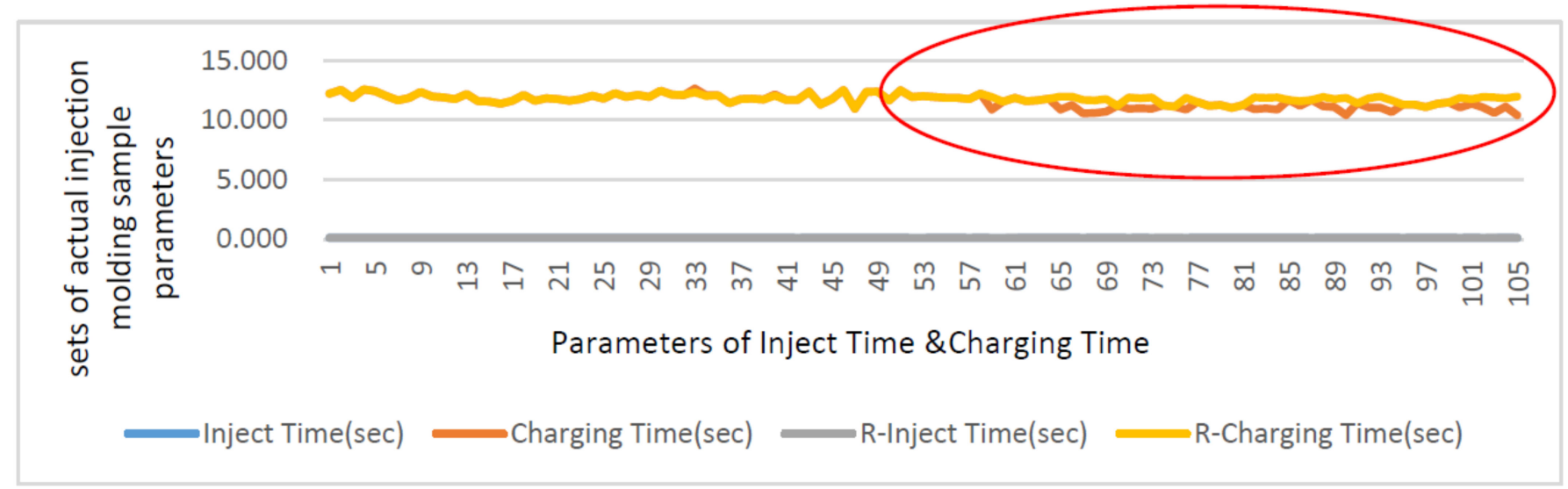

Figure 9. VM results of three cases: (1) without Step_Time; (2) with Step_Time; and (3) without Step_Time but with the assistance of the TVA method for the dry-etching process.

\section{Conclusions}

This paper uses AVM along with RPE to accommodate the production and adjustment process of the screw design unit. The RPE method aims to enhance the basic definition function of AVM to both automatically and quickly realize the screw groove in the screw prediction design goal. However, the purpose of the RPE method is not to replace the key features related to the machine center, but to quickly compensate for the TV gap between the method sample and the running sample when the different key features of the design TVs of the screw groove cannot be obtained. The example showed that the AVM and RPE methods are feasible and promising to meet the machine center production requirements for various injection screw industrial applications.

The TVA is used to determine when to stop executing the AVM method; usually, this value varies depending on the settings of the polymer materials used, but before the actual processing, the intelligent design AVM and RPE methods in this paper can be implemented. At the same time, the need for multiple trial adjustments to verify the accuracy of new samples in the future are reduced. This method can be said to be a new automatic virtual recognition concept as it does not consume actual processing energy and it will continue to be executed automatically until good accuracy is obtained.

Author Contributions: Conceptualization, H.-J.C.; Data curation, Z.-M.S.; Methodology, H.-J.C. and Z.-F.M.; Project administration, G.-Y.Z.; Writing—original draft, H.-J.C. All authors have read and agreed to the published version of the manuscript.

Funding: This research was funded by the 2019 Guangdong Province Science and Technology Special Fund Project—Major Science and Technology Special Project (2019ST102).

Conflicts of Interest: The authors declare no conflict of interest. 


\section{References}

1. Chen, D.-C.; Chen, T.-H.; Lin, G.-F.; Wang, Y.-K.; Chang, Y.-C. Experimental investigation into suitable process conditions for plastic injection molding of thin-sheet parts. Int. J. Eng. Technol. Innov. 2014, 4, 107.

2. Cheng, F.-T.; Huang, H.-C.; Kao, C.-A. Developing an automatic virtual metrology system. IEEE Trans. Autom. Sci. Eng. 2011, 9 , 181-188. [CrossRef]

3. Tieng, H.; Chen, C.-F.; Cheng, F.-T.; Yang, H.-C. Automatic virtual metrology and target value adjustment for mass customization. IEEE Robot. Autom. Lett. 2016, 2, 546-553. [CrossRef]

4. Lin, C.-Y.; Hsieh, Y.-M.; Cheng, F.-T.; Huang, H.-C.; Adnan, M. Time Series Prediction Algorithm for Intelligent Predictive Maintenance. IEEE Robot. Autom. Lett. 2019, 4, 2807-2814. [CrossRef]

5. Chiu, Y.-C.; Cheng, F.-T.; Huang, H.-C. Developing a factory-wide intelligent predictive maintenance system based on Industry 4.0. J. Chin. Inst. Eng. 2017, 40, 562-571. [CrossRef]

6. Yang, H.-C.; Tsai, T.-H.; Tieng, H.; Cheng, F.-T. A Gradual Refreshing Scheme for Improving Tool Utilization. IEEE Robot. Autom. Lett. 2019, 4, 515-522. [CrossRef]

7. Lin, Y.C.; Hung, M.H.; Huang, H.C.; Chen, C.C.; Yang, H.C.; Hsieh, Y.S.; Cheng, F.T. Development of advanced manufacturing cloud of things (AMCoT)—A smart manufacturing platform. IEEE Robot. Autom. Lett. 2017, 2, 1809-1816. [CrossRef]

8. Hung, M.-H.; Li, Y.-Y.; Lin, Y.-C.; Wei, C.-F.; Yang, H.-C.; Cheng, F.-T. Development of a novel cloud-based multi-tenant model creation service for automatic virtual metrology. Robot. Comput. Integr. Manuf. 2017, 44, 174-189. [CrossRef]

9. Yang, H.-C.; Li, Y.-Y.; Hung, M.-H.; Cheng, F.-T. A cyber-physical scheme for predicting tool wear based on a hybrid dynamic neural network. J. Chin. Inst. Eng. 2017, 40, 614-625. [CrossRef]

10. Yang, H.-C.; Tieng, H.; Cheng, F.-T. Automatic virtual metrology for wheel machining automation. Int. J. Prod. Res. 2016, 54, 6367-6377. [CrossRef]

11. Cheng, F.-T.; Hsieh, Y.-S.; Chen, C.-F.; Lyu, J.-R. Automated sampling decision scheme for the AVM system. Int. J. Prod. Res. 2016, 54, 6351-6366. [CrossRef]

12. Chen, C.-C.; Hung, M.-H.; Suryajaya, B.; Lin, Y.-C.; Yang, H.-C.; Huang, H.-C.; Cheng, F.-T. A Novel Efficient Big Data Processing Scheme for Feature Extraction in Electrical Discharge Machining. IEEE Robot. Autom. Lett. 2019, 4, 910-917. [CrossRef]

13. Lin, C.-Y.; Hsieh, Y.-M.; Cheng, F.-T.; Yang, Y.-R.; Adnan, M. Interaction-Effect Search Algorithm for the KSA Scheme. IEEE Robot. Autom. Lett. 2018, 3, 2778-2785. [CrossRef]

14. Cheng, F.-T.; Lin, C.-Y.; Chen, C.-F.; Xiao, R.-X.; Zheng, J.-W.; Hsieh, Y.-S. Blind-stage search algorithm for the key-variable search scheme. IEEE Robot. Autom. Lett. 2017, 2, 1840-1847. [CrossRef]

15. Yang, H.-C.; Li, Y.-Y.; Wu, M.-N.; Cheng, F.-T. A hybrid tool life prediction scheme in cloud architecture. In Proceedings of the 2016 IEEE International Conference on Automation Science and Engineering (CASE), Fort Worth, TX, USA, 21-24 August 2016; IEEE: Piscataway, NJ, USA, 2016; pp. 1160-1165.

16. Yang, H.-C.; Tieng, H.; Cheng, F.-T. Total precision inspection of machine tools with virtual metrology. J. Chin. Inst. Eng. 2016, 39, 221-235. [CrossRef]

17. Cheng, F.-T.; Tieng, H.; Yang, H.-C.; Hung, M.-H.; Lin, Y.-C.; Wei, C.-F.; Shieh, Z.-Y. Industry 4.1 for wheel machining automation. IEEE Robot. Autom. Lett. 2016, 1, 332-339. [CrossRef]

18. Yang, H.-C.; Adnan, M.; Huang, C.-H.; Cheng, F.-T.; Lo, Y.-L.; Hsu, C.-H. An Intelligent Metrology Architecture With AVM for Metal Additive Manufacturing. IEEE Robot. Autom. Lett. 2019, 4, 2886-2893. [CrossRef]

19. Tieng, H.; Tsai, T.-H.; Chen, C.-F.; Yang, H.-C.; Huang, J.-W.; Cheng, F.-T. Automatic virtual metrology and deformation fusion scheme for engine-case manufacturing. IEEE Robot. Autom. Lett. 2018, 3, 934-941. [CrossRef]

20. Cheng, F.-T.; Hsieh, Y.-S.; Zheng, J.-W.; Chen, S.-M.; Xiao, R.-X.; Lin, C.-Y. A scheme of high-dimensional key-variable search algorithms for yield improvement. IEEE Robot. Autom. Lett. 2016, 2, 179-186. [CrossRef]

21. Hsieh, Y.-M.; Lin, C.-Y.; Yang, Y.-R.; Hung, M.-H.; Cheng, F.-T. Automatic Virtual Metrology for Carbon Fiber Manufacturing. IEEE Robot. Autom. Lett. 2019, 4, 2730-2737. [CrossRef]

22. Chang, H.-J.; Chen, S.-L.; Lee, P.-Y. Direct cutting of a pyramid part to evaluate a five-axis machine tool with the Taguchi method. Int. J. Adv. Manuf. Technol. 2017, 91, 315-325. [CrossRef]

23. Chang, H.-J.; Chen, S.-L.; Lee, P.-Y. Applying a pyramid part in the performance evaluation of multiple types of five axis machine tools. The Int. J. Adv. Manuf. Technol. 2017, 92, 2247-2253. [CrossRef]

24. Chang, H.-J. Using a Cone Frustum Part in the Evaluation of Multiple Types of Five-Axis Machine Tools with the Taguchi Method. In Proceedings of the 2018 IEEE International Conference on Advanced Manufacturing (ICAM), Yunlin, Taiwan, 16-18 November 2018; IEEE: Piscataway, NJ, USA, 2018; pp. 209-212.

25. Chang, H.-J. Performance Evaluation of Multi-type Five-axis Machine Tool with Recognizable Performance Evaluation by Fuzzy Theory. In Proceedings of the 2019 Prognostics and System Health Management Conference (PHM-Qingdao), Qingdao, China, 25-27 October 2019; IEEE: Piscataway, NJ, USA, 2019; pp. 1-5.

26. Chang, H.-J.; Su, Z.-M. Optimizing Glass Fiber Molding Process Design by Reverse Warping. Materials 2020, 13, 1151. [CrossRef] [PubMed]

27. Castéran, F.; Ibanez, R.; Argerich, C.; Delage, K.; Chinesta, F.; Cassagnau, P. Application of Machine Learning Tools for the Improvement of Reactive Extrusion Simulation. Macromol. Mater. Eng. 2020, 305, 2000375. [CrossRef] 
28. Tadmor, Z. Fundamentals of plasticating extrusion. In Polymer Engineering \& Science; John Wiley \& Sons, Inc.: Hoboken, NJ, USA, 1966.

29. Tadmor, Z.; llan, J.D.; Klein, L. Melting in Plasticating Extruders Theory and Experiments. In Polymer Engineering E Science; John Wiley \& Sons, Inc.: Hoboken, NJ, USA, 1967. 